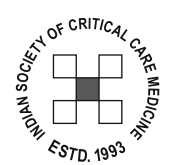

\title{
Critical care delivery in intensive care units in India: Defining the functions, roles and responsibilities of a consultant intensivist
}

\section{Recommendations of the Indian Society of Critical Care Medicine Committee on Defining the Functions, Roles and Responsibilities of a Consultant intensivist Members}

\author{
J. V. Divatia1, A. K. Baronia ${ }^{2}$, A. Bhagwati ${ }^{1}$, R. Chawla ${ }^{3}$, S. Iyer $^{4}$, C. K. Jani ${ }^{1}$, S. Joad ${ }^{5}$, V. Kamat ${ }^{6}$, \\ F. Kapadia ${ }^{1}$, Y. Mehta ${ }^{3}$, S. N. Myatra ${ }^{1}$, S. Nagarkar ${ }^{1}$, V. Nayyar ${ }^{7 *}$, S. Padhy ${ }^{8}$, R. Rajagopalan $^{6}$, \\ N. Ramakrishnan ${ }^{6}$, B. Ray ${ }^{9}$, S. Sahu ${ }^{10}$, S. Sampath ${ }^{11}$, S. Todi ${ }^{12}$
}

\section{Background}

Over the last few years, there has been a tremendous increase in the knowledge, technology and skills required to treat critically ill patients. This has lead to the development of intensive care units (ICUs), which are essentially areas, where severely ill patients can be concentrated and looked after and provided with the infrastructure and expertise necessary to treat critical illness. Current ICU patterns result in the patient being admitted under a primary consultant, before admission to the ICU. Often this primary consultant is not an intensive care specialist and may not be conversant with the current critical care practices and guidelines. The presence of a consultant intensivist (critical care specialist) in this setting has been demonstrated, to greatly increase patient safety and to improve outcomes in terms of morbidity, mortality, length of stay and costs.

While the concept of the intensive care unit has gained widespread acceptance amongst medical professionals, hospital administrators and the general public, recognition of the need and role for doctors specialising in intensive care medicine, has lagged behind. One of

\footnotetext{
From:

*(Formerly from Bangalore), 'Mumbai, ' 2 Lucknow, ${ }^{3}$ Delhi, ${ }^{4}$ Pune, 5 Jaipur,

${ }^{6}$ Chennai, ${ }^{7}$ Sydney, ${ }^{8}$ Indore, ${ }^{9}$ Jamshedpur, ${ }^{10}$ Bhubaneshwar, ${ }^{11}$ Bangalore, ${ }^{12}$ Kolkata
}

the reasons may be that intensive care medicine is a relatively new speciality; social, political and economic factors also undoubtedly play a role in preventing wholehearted acceptance of a consultant intensivist in the hospital. Nevertheless, it is becoming increasingly clear that the presence of an intensive care medicine specialist working in the intensive care unit improves outcomes, in terms of various previously mentioned quality indicators. ${ }^{[1]}$ In the United States, the Leapfrog Group, a group of more than 170 companies and organizations that buy health care and work together to reduce preventable medical mistakes and improve the quality and affordability of health care, mandates that hospitals fulfilling the ICU Physician Staffing standard will operate adult and/or pediatric ICUs that are managed or co-managed by intensivists. ${ }^{[2]}$

A number of our members are practicing as consultant intensivists on a part time or full time basis. Also, a number of trainees and graduates of the Indian Society of Critical Care Medicine (ISCCM) Certificate Course, the Post-Doctoral Fellowship programme of the National Board of Examinations (NBE) and other critical care programmes are being appointed as consultant intensivists in various hospitals throughout the country and this trend is going to increase. It is essential that the training, talent and services of trained consultant

Free full text available from www.ijccm.org 
intensivists be appropriately utilised in hospitals for the benefit of patients.

It is imperative that in this dual consultant model (involving the primary consultant and the consultant intensivist in the ICU), the relationship should be one that promotes an equal partnership and avoids conflict.

This committee of the ISCCM was set up to formulate recommendations on how consultant intensivists may be utilised in hospitals, so that they give their best as intensive care specialists and receive due recognition and acknowledgment of their efforts from patients and health care professionals.In this document, the terms intensive care medicine and critical care medicine and the terms consultant intensivist and critical care specialist, have been used interchangeably.

\section{Methods}

Committee members were selected to represent a variety of intensive care practices across the country. The experience of the committee member's practice ranged from practice in open, transitional and closed ICUs; senior, established consultant intensivists, to freshly qualified, young consultant intensivists; practice in large multispeciality hospitals to practice in nursing homes; practice in large cities to practice in smaller towns; private hospitals to public hospitals and academic centres. Individual committee members wrote of the systems they had adopted in their ICUs and hospitals, successes and failures of their respective systems of care and suggestions for improvement. The collective experiences were pooled towards development of the recommendations.

The Committee tried to answer the following questions: ${ }^{[3]}$

- Who is a consultant intensivist?

- Does a consultant intensivist make a difference to outcomes of critically ill patients?

- What system of ICU care is best?

- What is the role of the consultant intensivist in a hospital? I.e. how should the consultant intensivist function in the ICU in general and his interaction and relationship with the primary consultant in particular.

- How can the services of the consultant intensivist be best utilised for improved patient outcomes?

In order to answer the above questions, the Committee members reviewed the relevant literature and also looked at guidelines of professional organisations, including those of the ISCCM, ${ }^{[3]}$ Society of Critical Care Medicine (USA $)^{[4]}$ and the Australia and New Zealand Joint Faculty of Intensive Care Medicine. ${ }^{[5]}$ Recommendations are based on the interpretation of the literature as well as the expert opinion of the committee, arrived at after mutual discussion, personally and by email.

Levels of Evidence are graded as follows: Level I: Large, randomized trials with clear-cut results; low risk of falsepositive or false-negative error Level II: Small, randomized trials with uncertain results; moderate to high risk of false-positive and/or false-negative error Level III: Nonrandomized, concurrent cohort comparisons, contemporaneous controls Level IV: Nonrandomized, historical cohort comparisons/controls and expert opinion Level V: Case series, uncontrolled studies and expert opinion

\section{Who is a consultant intensivist?}

The ISCCM defines an intensivist as follows: The intensivist should have a postgraduate qualification* in Internal Medicine, Anaesthesia, Pulmonary Medicine or Surgery and either a) An additional qualification in Intensive Care such as DM, DNB Post Doctoral Fellowship, Certificate Course of the ISCCM, or qualifications from abroad such as the European Diploma in Intensive Care, American Board Certification, Australian or New Zealand Fellowship (FANZCA or FFICANZCA). OR b) At least one-year's training in a reputed ICU abroad. [*: A few candidates of the ISCCM Certificate Course who have been certified with a 3-year training programme in Intensive Care after M.B.B.S, are also recognised as Intensivisits. Since January 2003, M.B.B.S. graduates are no longer be eligible for certification and only those with a postgraduate diploma or degree can qualify for the course.]

In addition, persons so qualified or trained must have at least two-years' experience in the ICU (at least 50\% time spent in the ICU), to work in a secondary level (Level 2) ICU and three-years experience to work in a tertiary level (Level 3) ICU.

In case of doctors not having either of the above mentioned qualifications or training, they should have extensive experience in intensive care in India, quantified 
as at least three years' experience in the ICU (at least $50 \%$ time spent in the ICU), for a Secondary level (Level

2) ICU and five years experience for a Tertiary level (Level 3) ICU.

Does a Consultant intensivist make a difference to outcomes of critically ill patients?

A number of observational studies ${ }^{[6-12]}$ suggest that ICU mortality and costs are lower with a consultant intensivist present in the ICU.

There are a number of small, nonrandomized studies primarily using historical controls (level IV) that support the presence of a consultant intensivist in the the ICU compared, with a prior model without a consultant intensivist. These studies were usually done when there was a change in ICU organizational structure, primarily the addition of a consultant intensivist. ICU outcome data (usually mortality) from a time period before the addition of the consultant intensivist are compared with data for a time period, after the addition of the consultant intensivist care delivery.

Pronovost PJ, Jenckes MW, Dorman T, et al: Organizational characteristics of intensive care units related to outcomes of abdominal aortic surgery. JAMA 1999; 281:1310-1317 (level III evidence)

This is a large observational, nonrandomized study, using contemporaneous controls. The study was done using the Maryland Health Discharge Data Set, with a focus on 2987 patients undergoing major abdominal aortic surgery. The authors showed that daily rounds in the ICU by an ICU physician were associated with reduced in-hospital mortality and specific postoperative medical complications. The magnitude of this mortality reduction was equivalent to that observed in other studies, that compared the skill (and surgical volume) of operating surgeons.

Hanson CW, Deutschman CS anderson HL, et al: Effects of an organized critical care service on outcomes and resource utilization: A cohort study. Crit Care Med 1999; 27:270-274 (level III evidence)

This study compared two different concurrent care models of surgical ICU patients. One group was managed exclusively by the critical care attending service and the other by the general surgical faculty and house staff. Despite higher severity of illness scores, the critical care patient group had shorter ICU lengths of stay, fewer days of mechanical ventilation, fewer arterial blood gases, fewer consultations, fewer complications, shorter hospital lengths of stay and fewer Medicare-adjusted charges.

Pronovost PJ, Angus DC, Dorman T, et al: Physician staffing patterns and clinical outcomes in critically ill patients: A systematic review. JAMA. 2002; 288:2151-6

This systematic review of the available literature regarding ICU physician staffing and outcomes, concluded that, there is a consistent finding of decreased mortality and length of stay with consultant intensivist presence.

Reynolds NH, Haupt MT, Thill-Baharozian MC, et al: Impact of critical care physician staffing with septic shock in a university hospital medical intensive care unit. JAMA 1988; 260:3446-3450 (Level IV evidence)

In a retrospective review of MICU records, twC consecutive 12-month periods of time were compared. During the first time period, the ICU was without critica care-trained faculty and during the second time period, the ICU was supervised by critical care-trained faculty. Severity of illness scores were comparable during the two time periods. Mortality was significantly decreased during the post critical care medicine time period.

Brown JJ, Sullivan G: Effect on ICU mortality of a full-time critical care specialist. Chest 1989; 96:127. 129 (level IV evidence)

A retrospective review was conducted of two time periods (consecutive years) in a MICU, before and after the addition of a trained critical care specialist (consultant intensivist). Despite similar severity of illness, the mortality rate was significantly lower during the consultant intensivist time period.

Manthous CA, Amoateng-Adjepong Y, Al-Kharrat T, et al: Effects of medical consultant intensivist on patient care in a community teaching hospital. Mayo Clin Proc 1997; 72:391-399 (level IV evidence)

This was a retrospective review of MICU patient admissions comparing two consecutive time periods before and after the addition of a medical consultant intensivist. Patient severity of illness was similar during the two time periods. Mortality for pneumonia, mean length of hospital stay and MICU stay, were all reduced 
after the addition of the medical consultant intensivist.

Blunt MC, Burchett KR: Out-of-hours consultant cover and case-mix-adjusted mortality in intensive care. Lancet 2000; 356:735-736 (level IV evidence)

A historical case control study examined standardized mortality ratios in 452 patients admitted to an ICU after a consultant intensivist joined the staff, compared with 372 patients before the consultant intensivist's arrival. Severity of illness scores were comparable in both groups; however, the standardized mortality ratio improved significantly in the consultant intensivist group (0.81 vs. 1.11 ; ratio, 0.73 [95\% confidence interval, 0.55 0.97]).

\section{What system of ICU care is best?}

There is much debate on who should admit and manage critically ill patients in the ICU. A Closed unit is one, where once a patient enters the ICU, primary care of the patient is transferred to the consultant intensivist. The consultant intensivist takes all the major decisions in the ICU, including admission to and discharge from the ICU. Once the patient goes out of the ICU, care of the patient is transferred back to the primary consultant.

An Open ICU is one in which any consultant may admit a patient to the ICU, with or without the knowledge or consent of the consultant intensivists. Often, such ICUs may not even have a consultant intensivist on their staff. The primary consultant remains in charge, makes all decisions regarding patient management, including whether or not a consultant intensivist's consultation is required. The consultant intensivist may be asked to look after only certain parts of patient management (e.g. ventilation). It is not uncommon to find such a patient being managed by a series of single-organ specialists (e.g. gastroenterologist, nephrologist, neurologist, cardiologist, etc). As with all other consultations, the consultant intensivist's recommendations may not be accepted by the primary consultant. The junior staff in the ICU (housemen, registrars) report on that patient to the referring consultant and not to the consultant intensivist.

A Semiclosed or Transitional unit is one which lies in between. There is a mandatory consult and daily rounds by the consultant intensivist, for all patients admitted in the ICU. The primary consultant as well as the consultant intensivist play a significant role in patient care. Orders are written by consultant intensivist or by primary team, in consultation with consultant intensivist. A large proportion of care is provided by the consultant intensivist and his team, in consultation with the primary consultant. There is now an increasing body of literature supporting the closed ICU systems, over open ICUs. ${ }^{[13-18]}$

Topeli A, Laghi F, Tobin MJ. Effect of closed unit policy and appointing an intensivist in a developing country. Crit Care Med. 2005; 33:299-306

This study from Turkey looked at the effect of changing over to a closed system in a medical ICU of a university hospital. Data were prospectively collected over 5 months before the policy change (open policy) and over an initial 6 months (early closed policy) and subsequent 12 months (late closed policy) after the policy change. Instituting a closed policy and simultaneously appointing a critical care specialist was associated with the admission of sicker patients and more frequent use of invasive procedures. Compared with open policy, patients were approximately 4.5 times more likely to survive their hospital stay during early closed policy $(P<0.001)$ and approximately five times more likely during late closed policy $(P<0.0001)$. Among patients receiving mechanical ventilation, hospital mortality was lower during the early $(57 \%)$ and late closed periods (59\%), than during open period (91\%; $P<0.01)$. The authors concluded that the dual strategy of closed policy and simultaneously appointing an intensivist fostered admission of sicker patients and improved the survival of patients requiring admission to an ICU of a developing country.

Carson SS, Stocking C, Podsadecki T, et al: Effects of organizational change in the medical intensive care unit of a teaching hospital: A comparison of 'open' and 'closed' formats. JAMA 1996; 276:322-328 (level III evidence)

This was a prospective cohort study which compared two consecutive time periods of ICU care. In the first period, there was an open ICU organizational structure, wherein critical care specialists consulted on all ICU patients and made recommendations, but the admitting attending physician retained primary responsibility for patient care. The second period had a closed format. The critical care attending physician assumed primary responsibility for all patient care and the admitting physician was a consultant. Despite significantly higher 
severity of illness scores during the closed ICU organization, the risk-adjusted mortality score was 0.78 , compared with 0.90 in the open ICU organization. Resource utilization did not increase during the closed unit structure, despite higher severity of illness.

Multz, AS, Chalfin DB, Samson IM, et al: A closed medical intensive care unit (MICU) improves resource utilization when compared with an open MICU. Am J Respir Crit Care Med 1998; 157:14681473 (level IV evidence)

A retrospective analysis of two time periods in one hospital was compared, as the ICU administrative structure changed from an open organizational structure to a closed one. In addition, another cohort of patients was prospectively analyzed, wherein one group from one hospital managed in an open ICU organizational structure, was compared with another group from another hospital managed in a closed ICU organizational structure (prospective analysis). Illness severity and primary diagnostic categories between groups, were comparable. ICU and hospital length of stay was less in closed units. An open ICU format was associated with greater mortality prediction.

Ghorra S, Reinert SE, Cioffi W, et al: Analysis of the effect of conversion from open to closed surgical intensive care unit. Ann Surg 1999; 229:163-171 (level IV evidence)

This is a retrospective review comparing two time periods (open unit vs. closed unit) in a surgical ICU. Mortality and overall complications were significantly higher in the open-unit group, compared with the closedunit group.

Cole L, Bellomo R, Silvester W, et al: A prospective, multicenter study of the epidemiology, management and outcome of severe acute renal failure in a "closed" ICU system. Am J Respir Crit Care Med 2000; 162:191-196 (level III evidence)

This was a prospective, observational study examining the outcome of acute renal failure requiring replacement therapy (severe acute renal failure), within closed ICU systems in Australia. The study was conducted over a 3month period in all nephrology units and ICUs in the state of Victoria, Australia. By using the SAPS II score or a recently validated renal-failure specific score, the predicted mortality for these patients was shown to be
$59 \%$. Actual mortality was $49.2 \%$. The authors concluded that patients with renal failure managed in closed ICU systems in Australia, had favorable outcomes compared with predicted mortality.

Knaus WA, Draper EA, Wagner DP, et al: An evaluation of outcome from intensive care in major medical centers. Ann Intern Med 1986; 104:410-418 (level III evidence)

This study is the post hoc analysis of the original Acute Physiology and Chronic Health Evaluation (APACHE) II database. This study was a large, nonrandomized observational study. There were 13 hospitals and 5,030 patients used to develop the APACHE II severity of illness system. The authors ranked ICUs by the actual or observed mortality and the predicted hospital deaths (standardized mortality ratio, SMR). When stratified by SMR, it was demonstrated that the best ICU was well organized, with protocols and policies including the canceling of elective operating room cases, if no beds were available. There were also a high proportion of bedside nurses who had master's degree. In addition, there were no interns (postgraduate year-1) in this unit. The lowest-ranked hospital did not have an organized medical program and had a substantial shortage of nursing. There was an atmosphere of distrust and there was no coordination of care. It is concluded that organized ICUs as defined in this review, had lower mortality.

There is increasing evidence that closed ${ }^{[13-18]}$ or transitional models ${ }^{[6-12]}$ have better outcomes and resource utilisation, than open ICUs. The ISCCM discourages the adoption or continuance of open ICUs. Our preference is for the closed model to be adopted in general medical-surgical, as well as speciality ICUs; however the transitional model is acceptable till closed units are established.

Executive Summary of Recommendations Defining the functions, role and responsibilities of the consultant intensivist

\section{Clinical management}

\section{Admissions to and discharges from the ICU}

The consultant intensivist must be informed of all admissions to the ICU. Preferably, no ICU admission should take place without the prior knowledge of the consultant intensivist and the decision to transfer the 
patient to the ICU should be taken after consultation with the consultant intensivist, or a designated member of the critical care team.

Discharge of a patient from the ICU should be with the knowledge of the consultant intensivist. The consultant intensivist should have the authority to discharge patients from the ICU, in order to accommodate new admissions.

Overall co-ordination about admissions to and discharges from the ICU, should be done by the intensive care medical team.

It is essential to prioritise admissions. The consultant intensivist is ideally placed to triage patients, prioritise admissions and maintain a waiting list. $\mathrm{He} /$ she is also best placed to select the patients most suitable for early discharge, to make a bed available for admitting a new critically ill patient. This will ensure that intensive care will be available to patients who most deserve and are most likely to benefit from intensive care.

\section{Responsibility of the consultant intensivist}

The consultant intensivist must communicate with the referring consultants and explain the priority for admission, in a logical and scientific manner. The presence of written protocols outlining how patient admissions will be prioritised, is helpful. When discharging a patient from the ICU, the consultant intensivist should be satisfied that the patient is suitable for transfer out of the ICU and should communicate with the referring consultant, the reasons for an early discharge, as well as any special instructions to be followed on the wards.

All patients admitted to the ICU must be seen by a consultant intensivist.

- It is mandatory to have a critical care consult, once the patient is in the ICU.

- Except in a closed ICU where the consultant intensivist and his team takes over care of the patient, the responsibility for patient management is shared between the consultant intensivist and the primary consultant. It is mandatory that the relation between ICU and external consultants is one of an equal partnership.

- The consultant intensivist must do daily rounds on all ICU patients. It is desirable to get radiology, microbiology, pharmacy and input from other disciplines, during these rounds. It is helpful if members from the primary consultant's team are present during the ICU rounds. During these rounds, the consultant intensivist needs to do the following.

- Be familiar with all relevant clinical aspects of the history and presentation.

- Do a focused and relevant clinical examination.

- Review all the radiology, laboratory and microbiology data. At this point, he/she needs to ensure that all recent test reports have been collected, seen and appropriate action has been taken.

- Review the input by all the specialists involved in the care of the patient. If needed, further opinions may be sought.

- Make a clear plan for the next 12-24 hours. This should include all aspects of management including, but not limited to major organ support, fluid therapy, nutrition and antibiotics. It should also specify the appropriate preventive measures including, but not limited to prevention of pressure sores and appropriate DVT and stress ulcer prophylaxis.

- Check that all prior jobs generated by previous rounds and by other consultants have been completed.

- Ensure clear documentation of Consultant and junior medical staff notes.

- It is desirable that all orders are written by the intensive care medical team.

- The consultant intensivist should periodically review the patient's progress and may make necessary changes in patient management.

- A member of the ICU team should accompany the primary consultant on his rounds, to facilitate coordination between the consultant intensivist and the primary consultant.

- Any change in patient management desired by the primary consultant, should take place after discussion and consultation with the consultant intensivist. All new orders should then be written by the intensive care medical team.

- Progress notes should be written by the intensive care medical team, as well as the primary consultant's and other consultants' teams.

- Common ICU procedures should be performed by the consultant intensivist,or by personnel designated by the consultant intensivist under his / her supervision.

- The consultant intensivist must be informed of any change in the patients' condition. This information 
should also be communicated to the primary consultant. Any emergency intervention should be performed by the intensive care team.

- The consultant intensivist or referring consultant may seek specialist consultations for the ICU patient, when required. The consultant intensivist must co-ordinate the advice of various consultants and devise a plan of management for the patient as a whole.

- The decision to transport a patient within the hospital or outside the hospital must be taken by the consultant intensivist, in consultation with the primary consultant. The risks and benefits of transport must be considered.

- It is the responsibility of the intensive care team to organise safe transport of the patient from the ICU and back to the ICU. The ISCCM guidelines on transport of the critically ill patient (under preparation) may be followed.

\section{Responsibility of the consultant intensivist}

A major responsibility of the consultant intensivist is to ensure that any conflict is avoided, without compromising safety standards and by ensuring that standard practice guidelines and protocols are instituted in the care of the patient. This necessitates adequate communication between ICU and external consultants. The consultant needs to ensure that all ICU procedures are done safely and competently. Teaching and training of junior medical staff is the responsibility of the consultant intensivist.

\section{Communication with patients and their families}

- The consultant intensivist must communicate with the patients and / or their family members, the nature and seriousness of the illness, the plan of management and the progress of the patient.

The consultant intensivist should discuss the patient's progress with the primary consultant and ensure that the patient and family are given accurate and uniform information by all medical and nursing staff.

\section{End of life decisions}

- The consultant intensivist should recognise that intensive care may be futile in certain situations and initiate end-of life discussion with the family, in coordination with the primary consultant. The consultant intensivist must ensure that the primary consultant and other medical and nursing staff are in broad agreement, that an end-of-life discussion should be initiated.

- The consultant intensivist should identify brain-dead patients and liase with the transplant co-ordinators where appropriate.

\section{Responsibility of the consultant intensivist}

It is vital that conflicting information regarding the patient's illness is not given by different medical teams. This creates an atmosphere of distrust and suspicion and is the basis of dissatisfaction and legal action. In the Indian multicultural context, the intensivist should be sensitive to social and cultural issues. The consultant intensivist is advised to read the end-of-life position statement published by the ISCCM.19

\section{Time Commitment to the ICU}

- The consultant intensivist must either be present in the ICU during daytime working hours or in the hospital and rapidly available to the ICU when required.

- In a department with more than one consultant, cross cover between consultants should be ensured. A consultant roster may be made for ICU rounds and cover, procedure supervision, CPR and Medical Emergency Team (MET) and out of hours telephonic consultation and cover.

- It is not mandatory for the consultant intensivist to be present in the ICU at night. However junior medical staff that can effectively interact with the consultant and carry out orders, must be present in the ICU. The consultant intensivist must be continuously available for consultation on phone and come into the ICU if needed.

- Hospital managements should ensure that adequate number of consultant intensivists are appointed to provide coverage at all times, while making provision for leave, conferences, illness and vacations.

\section{Responsibility of the consultant intensivist}

There is a need for the presence of trained intensive care medical staff round the clock. It is the duty of the consultant intensivist to appoint, train and teach junior medical staff and roster them so that the ICU is staffed by competent personnel, especially outside routine hours. Written protocols should be available for patient management, including but not limited to patient assessment, procedures, management of common 
emergencies and transport.

\section{Privileges Outside the ICU}

The consultant intensivist may undertake commitments outside the ICU (e.g. clinics, admission privileges in the ICU and wards), but must ensure that he / she is readily available to the ICU at all times.

\section{Services outside the ICU}

- The consultant intensivist may also provide services such as but not limited to

- Management of patients in the high-dependency unit

- Medical Emergency Team:

- Emergency department, including the Trauma team

- CPR team

- Artificial airway management

- Invasive procedures outside the ICU (e.g, Central Line, PICC lines, Lumbar puncture, Insertion of feeding tubes etc)

A consultant intensivist may act as a consultant for the ICU Outreach Services. The outreach services of critical care medicine plays a pivotal role in improving patient care, in wards by providing all logistic support for early recognition of acute life threatening events and treatment interventions to stabilize sick patients who are at the risk of developing life threatening events. The concept of the medical emergency team (MET) is gaining wide acceptance.

\section{Follow-up of patients outside the ICU}

- Once a patient has been discharged from the ICU, the consultant intensivist must transfer care to the primary consultant.

- The consultant intensivist may continue to provide care to the patient on the wards, if requested to do so by the primary consultant

- If the consultant intensivist is the primary consultant of the patient, then the above do not apply.

\section{Maintaining standards of intensive care practice}

- One of the major roles of the consultant intensivist is to ensure that adequate medical and safety standards are maintained throughout the patients stay. This is best done by the creation and implementation of appropriate protocols and practice guidelines. These include, but are not limited to, protocols for

- Performance of standard ICU procedures
- Mechanical ventilation, renal replacement therapy and other major life support

- Care of the airway

- Sedation and analgesia

- Infection control

- Transport of the ICU patient

- It is the duty of the consultant intensivist to ensure that the nursing, technical and junior medical staff,are aware of the protocols and comply with their implementation.

- Maintenance of a clear chain of command is essential for optimal patient care. It is the duty of the consultant intensivist to ensure that major decisions are not made independently by junior medical staff. Its is equally the duty of the consultant intensivist to ensure that major decisions are not unduly delayed, due to inability to contact a consultant. Protocolized care and availability of second on call consultant intensivists should ensure that safety standards are not compromised.

\section{Administration}

- When more than one consultant intensivist is appointed in the ICU, it is highly desirable that that they function as a department, rather than autonomous individuals.

- In such a department, one of the consultant intensivists should function as the ICU Director.

- The consultant intensivist should co-ordinate with other departments in the hospital, to ensure that their services are delivered to the ICU in an efficient and timely manner, whenever required.

- The consultant intensivist must play a key role in the design, maintenance and upgradation of the ICU, including the physical structure and equipment in the ICU.

- The consultant intensivist should have a clear role in the choice of equipment, drugs and other consumables like catheters, filters etc. He / she must firmly veto the purchase and use of substandard products, in an attempt at cost saving or profit maximizing.

- The consultant intensivist should interact with the hospital administration to ensure that the ICU is staffed with adequate number of appropriately qualified staff, including junior medical staff, nurses and other paramedical staff.

- The consultant intensivist should maintain unit 
statistics and run regular audits and quality assurance and quality improvement programmes for the ICU.

- The consultant intensivist must be member of the hospital infection control committee, purchase committee and play a role in the formulation of antibiotic policies, transport policies, admission and discharge policies, etc.

\section{Payments}

- In the interest of transparency and justice, all payments should be documented and be of a rationalized structure

- These should be in accordance with hospital policy and in accordance with the underlying principle of an at par relationship with other consultants. The consultant intensivist may receive a fixed salary, or a fee for service.

- If fee is for service, the fees include consultation charges, which may be more than one in a day and procedure charges.

- Charges for procedures performed by the ICU team in the ICU should be billed to the consultant intensivist. All charges should be at par with similar services provided by other speciality consultants, or departments.

\section{Teaching}

- Teaching forms a vital component of the consultant intensivist's workload. Even in units that do not have formal training programmes, it is essential that junior medical staff is taught the basics of intensive care. This is because medical graduates have had little or no exposure to intensive care during their undergraduate or postgraduate training. Junior medical staff should be taught and encouraged to attend courses including, but not limited to Basic and Advanced Cardiac Life Support, Acute Trauma Life Support and Fundamentals of Critical Care Support. They should be made familiar with management guidelines for common problems, including but not limited to management of acute myocardial infarction and the Surviving sepsis guidelines. Procedures should be taught and supervised and emphasis must be laid on infection control measures, including handwashing, aseptic techniques and rational prescription of antibiotics.

- Training programmes and protocols for nurses should be developed.
- It is also useful to conduct symposia and case discussions in the hospital to increase awareness and confidence in the capabilities of the intensive care unit.

\section{Role of the Consultant Intensivist in a Teaching Hospitals}

- Although true for almost all clinical specialties, a teaching ICU more than any other, requires an environment where a teacher assumes the responsibility of role modelling. This is especially important not only in how their ICU trainees are moulded, but also how other residents training in other specialities look at consultant intensivists and ICUs.

- Teaching hospitals should follow as much of a closed model as possible.

- Courses covering concepts of intensive care medicine may be offered to postgraduate students of various faculties. The courses can teach

- Cardiopulmonary resuscitation

- Airway obstruction: Early recognition and intervention

- Circulatory instability: Early recognition and intervention

- General Principals of Mechanical Lung Ventilation o General Principals of Intensive Care

- Prevention of Acute Life Threatening Events in patients admitted in wards

\section{Research}

- Consultant intensivists are encouraged to conduct research, especially in topics relevant to India

- Consultant intensivists should also participate in acquisition of Indian data relating to global issues in critical care.

- Research may take the form of audits, observational studies and randomised clinical trials

- Audits are valuable for both quality improvement, as well as research projects.

- Consultant intensivists should follow standard ethical and regulatory guidelines in the conduct of research.

- There is paucity of data with the ISCCM on the current models of delivery of intensive care and the current role and status of intensivists across the country. The committee recommends that the ISCCM should maintain a database of ICU's and survey delivery models and intensive care practice every year. This will not only provide invaluable epidemiological data, but will help track the changing patterns of intensive 
care delivery in India.

- The ISCCM should consider evolving a mechanism by which patient data (including progress notes and ICU flow-charts) are recorded in a standardised uniform format. All ICUs should also be encouraged to maintain data on admissions and have some scoring system in place. This will facilitate capturing of data on admissions diagnoses, disease patterns, case-mix and epidemiology of intensive care medicine in India. In the long-term, this will help in planning future multi-centre trials in Indian ICUs.

\section{General Responsibilities of the Consultant Intensivist}

- In the hospital, the consultant intensivist must demonstrate ethical values, expertise and professionalism, in day-to-day work. The consultant intensivist must put the patient at the centre of all decisions and actions.

- The consultant intensivist should promote the culture of competent, compassionate and cost-effective care of the critically ill patient

- The consultant intensivist should develop and maintain excellent rapport, co-ordination and communication with various colleagues, administrators and other hospital personnel to facilitate high quality patient care.

- The consultant intensivist should evolve a work culture that promotes relationships and avoids conflict.

- The consultant intensivist should continuously upgrade his / her knowledge, competence and skills. This will not only benefit patient care, but also improve confidence in the consultant intensivist and the ICU

- The consultant intensivist should work towards creating awareness of the speciality of intensive care medicine amongst the medical profession, as well as the general public.

\section{References}

1. The Leapfrog Group. ICU Physician staffing (IPS) Bibliography. http://www.leapfroggroup.org/for_hospitals/ leapfrog_safety_practices/icu_physician_staffing/ ips_bibliography. Accessed on 06/07/2005.

2. The Leapfrog Group for patient safety. Factsheet: ICU Physician Staffing. accessed from http://www.leapfroggroup.org/media/file/ Leapfrog-ICU_Physician_Staffing_Fact_Sheet.pdf Accessed on 06/07/2005.
3. Indian Society of Critical Care Medicine. Indian Guidelines for Critical Care Units. February 10, 2003.

4. Brilli RJ, Spevetz A, Branson RD, Campbell GM, Cohen H, Dasta JF, et al. American College of Critical Care Medicine Task Force on Models of Critical Care Delivery. The American College of Critical Care Medicine Guidelines for the Definition of an Intensivist and the Practice of Critical Care Medicine. Critical care delivery in the intensive care unit: defining clinical roles and the best practice model. Crit Care Med 2001;29:2007-19.

5. Faculty of Intensive Care, Australia and New Zealand College of Anaesthetists. The Duties of an intensive care specialist in hospitals accredited for intensive care training. http:// www.jficm.anzca.edu.au/publications/policy/ic2_2000.htm Accessed on 06/07/2005

6. Pronovost PJ, Jenckes MW, Dorman T, Garrett E, Breslow MJ, Rosenfeld BA, et al. Organizational characteristics of intensive care units related to outcomes of abdominal aortic surgery. JAMA 1999;281:1310-7.

7. Hanson CW $3^{\text {rd }}$, Deutschman CS, Anderson HL $3^{\text {rd }}$, Reilly PM, Behringer EC, Schwab CW, et al. Effects of an organized critical care service on outcomes and resource utilization: A cohort study. Crit Care Med 1999;27:270-4.

8. Pronovost PJ, Angus DC, Dorman T, Robinson KA, Dremsizov TT, Young TL. Physician staffing patterns and clinical outcomes in critically ill patients: a systematic review. JAMA 2002;288:215162.

9. Reynolds NH, Haupt MT, Thill-Baharozian MC, Carlson RW. Impact of critical care physician staffing with septic shock in a university hospital medical intensive care unit. JAMA 1988;260:3446-50.

10. Brown JJ, Sullivan G. Effect on ICU mortality of a full-time critical care specialist. Chest 1989;96:127-9.

11. Manthous $\mathrm{CA}$, Amoateng-Adjepong $\mathrm{Y}$, al-Kharrat $\mathrm{T}$, Jacob $\mathrm{B}$, Alnuaimat HM, Chatila W, et al. Effects of medical consultant intensivist on patient care in a community teaching hospital. Mayo Clin Proc 1997;72:391-9.

12. Blunt MC, Burchett KR. Out-of-hours consultant cover and casemix-adjusted mortality in intensive care. Lancet 2000;356:7356.

13. Topeli A, Laghi F, Tobin MJ. Effect of closed unit policy and appointing an intensivist in a developing country. Crit Care Med 2005;33:299-306.

14. Carson SS, Stocking C, Podsadecki T, Christenson J, Pohlman A, MacRae S, et al. Effects of organizational change in the medical intensive care unit of a teaching hospital: A comparison of 'open' and 'closed' formats. JAMA 1996;276:322-8.

15. Multz, AS, Chalfin DB, Samson IM, Dantzker DR, Fein AM, Steinberg $\mathrm{HN}$, et al. A closed medical intensive care unit (MICU) 
improves resource utilization when compared with an open MICU. Am J Respir Crit Care Med 1998;157:1468-73.

16. Ghorra S, Reinert SE, Cioffi W, Buczko G, Simms HH. Analysis of the effect of conversion from open to closed surgical intensive care unit. Ann Surg 1999;229:163-71.

17. Cole L, Bellomo R, Silvester W, Reeves JH. A prospective, multicenter study of the epidemiology, management and outcome of severe acute renal failure in a "closed" ICU system. Am J Respir
Crit Care Med 2000;162:191-6.

18. Knaus WA, Draper EA, Wagner DP, Zimmerman J. An evaluation of outcome from intensive care in major medical centers. Ann Intern Med 1986;104:410-8.

19. Mani RK, Chawla R, Divatia JV. Guidelines for limiting lifeprolonging interventions and providing palliative care towards the end of life in Indian Intensive care units. Indian J Crit Care Med 2005;9:96-107. 